\title{
Frailty, Thy Diagnosis Is Uncertain: Impact on IBD Readmission and Mortality
}

\author{
Simon J. Hong ${ }^{1} \cdot$ Seymour Katz ${ }^{2}$ \\ Accepted: 23 February 2021 / Published online: 22 March 2021 \\ (c) The Author(s), under exclusive licence to Springer Science+Business Media, LLC, part of Springer Nature 2021
}

The term frailty was first used in 1979 to explain the heterogeneity in mortality of individuals, with its main focus on the decline in fitness and nutritional status associated with aging $[1,2]$. Frailty is now understood to be a multisystem dysregulation of homeostatic reserves that can occur at any age. This is characterized by increased vulnerability to stressors resulting from deficits in multiple physiologic systems, i.e., the immune, musculoskeletal, endocrine, and neurologic systems [3].

Frailty has been associated with poor outcomes of numerous chronic disease states, including cirrhosis, heart failure, chronic kidney disease, diabetes, and also in patients undergoing surgery [4]. Given that inflammatory bowel disease (IBD) is a disorder of chronic inflammation and immune dysregulation, patients with IBD may be particularly at risk for frailty [5]. Despite the growing awareness of frailty, it remains underdiagnosed in patients with IBD. In one cohort study of 135 patients with IBD aged 65 or older who were screened for frailty, $23 \%$ of patients had increased vulnerability and 44\% had impairment [6].

Much of the existing data on frailty in IBD have been published using large healthcare databases and diagnosis codes to identify frail patients. In a study of 11,000 patients with IBD in the Partners Healthcare System, Kochar et al. [7, 8] used the Frailty Risk Score (FRS), an algorithm of ICD-10 diagnosis codes, in order to identify frailty traits and found that frailty was associated with a two-fold increase in

Simon J. Hong

simon.hong@nyulangone.org

Seymour Katz

seymourkatz.md@gmail.com

1 Division of Gastroenterology and Hepatology, Inflammatory Bowel Disease Center at New York University Langone Health, 305 East 33rd Street, New York, NY, USA

2 Division of Gastroenterology and Hepatology, Inflammatory Bowel Disease Center at New York University Langone Health, 1999 Marcus Ave, New Hyde Park, New York, NY, USA treatment-related infections and a three-fold increase in mortality, independent of age. Qian et al. [9] used the National Readmissions Data (NRD) database, a representative sample of US hospitalizations, to evaluate 47,402 patients hospitalized in 2013 , finding that $33 \%$ of patients were frail as defined by the FRS, with an associated $57 \%$ increase in mortality rate and $21 \%$ increase in risk of readmission.

In this issue of Digestive Diseases and Sciences, Faye et al. [10] add to this growing body of literature by reporting the largest representative sample of frailty in patients with IBD to date. The authors employ an alternative method to identify frailty, the Johns Hopkins Adjusted Clinical Groups-diagnosis predictive modeling (ACG Dx-PM) which has been validated to show moderate concordance with the Vulnerable Elders Survey (VES) scale in predicting adverse outcomes. This model assesses diagnoses from multiple domains of frailty, including malnutrition, dementia, visual impairment, urinary or fecal incontinence, weight loss, social support needs, and physical disability.

Analyzing the NRD database from 2010 to 2014, Faye et al. [10] evaluated 1,405,529 index admissions, finding that the prevalence of frailty rose from $10.2 \%$ in 2010 to $11.5 \%$ in 2014 , likely due to an aging IBD population. Frailty was an independent predictor of 30-day readmission (adjusted RR $1.15,95 \%$ CI 1.14-1.17) and 30-day readmission mortality (adjusted RR 1.12, 95\% CI 1.02-1.23) after multivariable adjustment for age, sex, co-morbidities, and surgical status. After excluding diagnoses in the frailty score that may be directly related to IBD disease activity, e.g., malnutrition, bowel incontinence and weight loss, frailty remained a significant negative predictor. Interestingly, increasing age was not an independent predictor of inferior outcomes, highlighting frailty as a more accurate predictor of adverse outcomes than biologic age. Frail patients had a mean longer length-of-stay (9.6 vs. 6.5 days) and higher hospitalization costs $(\$ 20,916$ vs. $\$ 13,539)$ when compared with non-frail patients (Table 1). 
Table 1 The burden and impact of frailty in patients with IBD

\begin{tabular}{ll}
\hline 1. & There is an increasing prevalence of frailty over time [10] \\
2. & Frailty is associated with an average 3-day longer hospital stay and 55\% higher hospitalization costs [10] \\
3. & Frailty is present in 11 to $33 \%$ of hospitalized patients with IBD [7, 9, 10] \\
4. & Frailty is associated with an increase in IBD treatment-related complications [8] \\
5. & Frailty is an independent risk factor for 30-day readmission and mortality, even after adjusting for age \\
a. & No prospectively validated frailty assessments yet exist for patients with IBD \\
\hline
\end{tabular}

Though the studies described above contribute important insights into the impact of frailty on outcomes in patients with IBD, several limitations exist. Since frailty scores such as the FRS and ACG Dx-PM have been validated in elderly patients but not specifically in patients with IBD, the precise definition of frailty remains undefined in this population. A limitation of retrospective studies using ICD codes is the risk of misclassification or underreporting as patients may lack appropriate coding documentation. Currently existing diagnosis codes may not accurately capture pre-frailty, in which accumulating deficits have not as yet manifested in any measurable declines in homeostatic function. Lastly, large databases such as the NRD do not contain granular data regarding IBD disease severity or activity, which are important contributors to the development and progression of frailty.

Therapeutic management of IBD has become increasingly more precise in the era of 'personalized medicine'. Frailty will become an important parameter in the risk stratification of patients with IBD. Before assessment of frailty can become a fully integrated into IBD patient care, several hurdles remain. Future prospective studies will need to identify frailty measurement tools in patients with IBD, which are both validated and feasible for widespread clinical use. The impact of frailty on therapeutic outcomes, and conversely the impact of control of IBD inflammation on frailty must be elucidated. Finally, targeted interventions in frail or prefrail patients need to be assessed in randomized controlled trials in order to identify the opportunities for changing the disease course in patients with IBD.

\section{References}

1. Vaupel JW, Manton KG, Stallard E. The impact of heterogeneity in individual frailty on the dynamics of mortality. Demography. 1979; 16:439-454
2. Rockwood K, Stadnyk K, MacKnight C. A brief clinical instrument to classify frailty in elderly people. Lancet. 1999;353:205-206

3. Clegg A, Young J, Iliffe S, Rikkert MO, Rockwood K. Frailty in elderly people. Lancet. 2013;381:752-762

4. Thompson $\mathrm{C}$, Taleban S. Incorporating frailty in the treatment program of elderly patients with gastrointestinal disease. Curr Treat Options Gastroenterol. 2020;18:635-656

5. Ramos GP, Papadakis KA. Mechanisms of disease: inflammatory bowel diseases. Mayo Clin Proc. 2019;94:155-165

6. Asscher V, Meijer L, Waars S, Slagboom J, Van der Meulen- de Jong A, Mooijaart S, et al. P732 Disability in older IBD patients. J Crohn's Colitis. 2018;12:S481-S482.

7. Kochar B, Cai W, Cagan A, Ananthakrishnan AN. Frailty is independently associated with mortality in 11001 patients with inflammatory bowel diseases. Aliment PharmacolTher. 2020;52:311-318

8. Kochar B, Cai W, Cagan A, Ananthakrishnan AN. Pretreatment frailty is independently associated with increased risk of infections after immunosuppression in patients with inflammatory bowel diseases. Gastroenterology. 2020;158:2104-2111

9. Qian AS, Nguyen NH, Elia J, Ohno-Machado L, Sandborn WJ, Singh S. Frailty is independently associated with mortality and readmission in hospitalized patients with inflammatory bowel diseases. Clin Gastroenterol Hepatol. 2020;S1542-3565(20)311198. [Epub ahead of print].

10. Faye AS, Wen T, Soroush A, Ananthakrishnan AN, Ungaro R, Lawlor $\mathrm{G}$, et al. Increasing prevalence of frailty and its association with readmission and mortality among hospitalized patients with IBD. Dig Dis Sci. (Epub ahead of print). doi: https://doi.org/10. 1007/s10620-020-06746-w.

Publisher's Note Springer Nature remains neutral with regard to jurisdictional claims in published maps and institutional affiliations. 